Cinémas

Revue d'études cinématographiques

Journal of Film Studies

\title{
Le Corps dansant au cinéma
}

\section{Ratiba Hadj-Moussa}

Volume 3, numéro 2-3, printemps 1993

URI : https://id.erudit.org/iderudit/1001199ar

DOI : https://doi.org/10.7202/1001199ar

Aller au sommaire du numéro

Éditeur(s)

Cinémas

ISSN

1181-6945 (imprimé)

1705-6500 (numérique)

Découvrir la revue

Citer cet article

Hadj-Moussa, R. (1993). Le Corps dansant au cinéma. Cinémas, 3(2-3), 205-221. https://doi.org/10.7202/1001199ar

\section{Résumé de l'article}

Cet article s'intéresse aux relations entre le cinéma et la danse en les concevant comme des rituels où se dit une certaine parole du social. Il prend appui sur le corps pour interroger les modalités qui rendent possible cette parole et qui participent à sa production. d'utilisation que vous pouvez consulter en ligne.

https://apropos.erudit.org/fr/usagers/politique-dutilisation/ 


\title{
Le Corps dansant au cinéma ${ }^{1}$
}

\section{Ratiba Hadj-Moussa}

\begin{abstract}
RÉSUMÉ
Cet article s'intéresse aux relations entre le cinéma et la danse en les concevant comme des rituels où se dit une certaine parole du social. Il prend appui sur le corps pour interroger les modalités qui rendent possible cette parole et qui participent à sa production.
\end{abstract}

\begin{abstract}
This article investigates the relationship between cinema and dance in an approach that sees both as rituals through which a particular social communication is expressed. Focusing on the body, it interrogates the modalities that make this communication possible, and that contribute to its production.
\end{abstract}

\section{Ce que danser au cinéma veut dire}

Bien que la danse et le cinéma soient associés depuis des décennies, peu d'études se sont arrêtées sur les significations auxquelles ils renvoient en tant que modes d'expression du social. Les travaux sur la danse et le cinéma se sont essentiellement intéressés à la comédie musicale en tant que genre. Parmi les travaux marquants, notons l'ouvrage de Rick Altman ${ }^{2}$, qui analyse l'évolution de la comédie musicale en démontrant ses mécanismes internes et en montrant comment elle sert de réceptacle et de point de fuite à une société américaine en perte de valeurs. Notons aussi le livre de Lucy Fischer ${ }^{3}$ qui montre, quant à lui, comment ce genre instaure un regard caméra (masculin) qui vise à assujettir les personnages féminins et à les réduire en purs objets de spectacle. Ces deux tentatives, qui 
retracent avec une rare finesse les grandes articulations de la comédie musicale, abordent allusivement la place et le statut du cinéma et de la danse dans une culture donnée.

Les analyses qui reposent sur le paradigme de la représentation - le cas de la quasi majorité des analyses filmiques - présupposent toujours un renvoi, aussi minime soitil, entre le social et les systèmes représentatifs, mais laissent ce renvoi obscur et inexpliqué.

Dans cet article, je me propose de comprendre ce renvoi à travers l'exemple de la danse et du cinéma, plus précisément du corps dansant au cinéma. J'émets l'hypothèse que la danse et le cinéma sont des rituels à travers lesquels se dit la parole du social. Ils ne se contentent pas de reproduire le social, ils le disent et l'actualisent de manière spécifique, c'est-à-dire à partir des matériaux qui leur sont propres. Autrement dit, on ne peut pas faire abstraction de la spécificité de leur langage et de leur technique. Ils ne sont pas des systèmes miniatures reproduisant indéfiniment la société (ils ne la miment pas simplement) mais des systèmes qui rendent possible la croyance en son existence; des relais qui construisent «la fiction» indispensable aux humains. Chaque culture négocie en effet sa continuité en fabriquant une sorte de lieu supra légitime qu'on appelle société ou tiers social ${ }^{4}$. La reconnaissance de ce tiers s'établit par l'action de ses médiations symboliques, en l'occurrence ici la danse et le cinéma, qui rendent compte à leur tour du faisable, du pensable et de l'acceptable, en un mot de la norme. Ces relais se situent dans une position intermédiaire dans la mesure où ils sont à la fois dans le social et en dehors de lui, en retrait ${ }^{5}$. La distanciation est indispensable à l'interprétation du social, à sa représentation qui est toujours symbolique et arbitraire. L'interprétation, qui mise sur le sens et la signification, repose sur la dimension du croyable auquel les sujets humains ne peuvent échapper.

Or toute symbolisation exige non seulement l'élaboration d'un langage (cinématographique par exemple), mais aussi des objets qui expriment le sens. Le corps en est un, car il est un des principaux lieux qui fait se recouper la danse et le cinéma. Corps comme carrefour et comme «signifiant flottant» (p. 94) selon l'expression de José Gil, sur lequel vient s'inscrire et se marquer l'ordre du social. Lorsque le corps est mis en jeu, lorsqu'il danse, il parle toujours de et pour quelque chose d'autre, notamment (et peut-être essentiellement) de la façon dont chaque société élabore sa propre rationalité (sa propre vérité et sa propre raison). 
L'ordonnancement et le montage auxquels le corps dansant est soumis remettent ainsi en cause la conception qui considère la danse comme un mode d'expression subversif6. La danse n'est pas en dehors du social, détachée de lui, parce qu'elle ne peut exister sans lui. Cet entre-deux qui la définit empêche de l'envisager comme une pratique radicalement subversive et libératrice, empoignant à elle seule la jouissance.

La psychanalyse nous apprend bien «qu'au delà de l'interdit, la transgression n'est rien» 7 . Inscrite dans le système et non en dehors de lui, la subversion impliquée dans la danse joue sur les limites du système et les signifie. Or que signifie jouer sur les limites si ce n'est mettre en relief, «en position détachée» (p. 144) selon les mots de Philippe Hamon, l'inscription d'une parole symbolique qui oriente et participe à l'élaboration de la subjectivité 8 , c'est-à-dire à la définition des sujets humains situés dans un ordre social donné. La danse et le cinéma servent tous deux de cadre à la définition du même et de l'autre et contribuent à la mise en place du principe de l'altérité, de la distance et de la séparation nécessaires et fondamentales à la constitution du sujet.

Dans la danse, l'institution de ce principe de division se fait paradoxalement sur un leurre, car à l'instar des corps en transe un corps qui danse se dédouble, se met hors de lui-même, fabrique un corps second qui laisse croire qu'il fait Un avec l'Idéal, c'est-à-dire avec le fondement. Or le fondement n'est fondement que parce qu'il échappe à la représentation, que parce qu'il nous échappe. Mais cela, nous feignons de l'ignorer 9. Dans la perspective psychanalytique qui est la sienne, Nelly Costelcalde écrit : «La danse serait l'expression et par là même, la résolution imaginaire d'une nostalgie de l'incorporation" (p. $\left.126^{10}\right)$. Imaginaire est bien le mot juste pour signifier que cette recherche se fait à l'insu du sujet, dont le corps est un corps qui ne sait pas, un corps fasciné en train de construire un autre corps qui, lui, sait ${ }^{11}$. Dans ce jeu de l'identification sidérante la danse, a fortiori lorsqu'elle est soutenue par la batterie des systèmes chorégraphiques est une parole silencieuse du corps, visible dans ses contorsions, qui à leur tour disent autre chose, précisément le dépassement de sa présence phénoménologique.

Ce long préambule sur la fonction et l'expressivité de la danse, plus exactement du corps dansant, montre que le cinéma ne se saisit pas de cet «objet» comme il le ferait des autres objets. Le corps est une sorte de topoi à partir duquel les systèmes représentatifs signifient leur inclusion dans le social et leur participation à son élaboration. Les lignes qui suivent abordent le traitement du corps dansant au cinéma comme un nœud où se 
projette un discours sur le social, et l'envisagent comme une modalité constitutive de l'organisation de ce discours. Cette dynamique est exemplifiée en trois moments : le niveau de ce que j'appelle la métamorphose, le dressage du corps dansant et, enfin, la beauté du corps dansant.

Neuf films serventt plus ou moins directement d'exemples : All That Jazz (Bob Fosse), Cabaret (Bob Fosse), Carmen (Carlos Saura), Cotton Club (Francis Ford Coppola), Dirty Dancing (Emile Adorlino), Flash Dance (Adrian Lyne), Isadora Duncan (Karel Riesz), Saturday Night Fever (John Badham), Soleil de nuit (Taylor Hackford).

\section{De la transformation}

L'état métamorphique du corps dansant trouve un point d'ancrage non négligeable au cinéma qui est aussi un art du double. Divers niveaux de l'écriture filmique soutiennent en effet la production / création de ce corps second. D'abord le dialogue. Dans Flash Dance la jeune danseuse de bar, qui souhaite devenir danseuse de ballet, confesse ainsi à son compagnon ses propres sensations lorsqu'elle danse :

Tu es sur scène, la musique attaque et tu commences à la sentir. Et puis, ton corps se met en mouvement. Je sais que ça a l'air d'être idiot, mais j'ai l'impression que quelque chose se déclenche. Je sens que je décolle, et puis, d'un seul coup, j'ai le sentiment d'être quelqu'un d'autre. Il y a des soirs où je ne peux pas attendre. Je suis impatiente d'être sur scène, rien que pour disparaître.

Disparaître pour se retrouver dans un lieu d'où il est possible de parler de l'Idéal, de déclamer son désir et d'oublier ce corps trop terrestre. Dans ces propos de la jeune danseuse, il y a réfutation de ce corps premier et métamorphose. Cette métamorphose s'articule au cinéma selon deux dimensions. La première se traduit, dans le cas de Flash Dance, dans la diégèse du film et dans la figuration, particulièrement à travers le striptease - dénudation quelque peu pudique - qui est, si l'on regarde bien la chose, prémisse à ce corps qui va disparaître et survoler les zones célestes. Cette dénudation-effeuillage à laquelle s'adonnent les danseuses est souvent elliptique (les gestes du déshabillage ne sont pas montrés). Elle est une véritable implosion qui dévoile et enlève progressivement non seulement l'enveloppe (l'habit), mais transforme aussi les personnages dansants (les danseuses changent d'apparence et même d'origine, passant du Japon à l'Occident — c'est-à-dire l'Amérique du Nord 
— en l'espace d'une seule danse) à tel point qu'ils ne sont plus qu'une réalité, qu'une forme-corps en mouvement.

À ce dépouillement s'ajoute, toujours dans cette première dimension de la métamorphose, la question de l'incarnation des personnages par les danseurs et les danseuses (Flash Dance, All That Jazz, Carmen, Dirty Dancing, Soleil de nuit...). Cette incarnation est complexifiée par des mises en abîme successives (ex. : des danseurs qui dansent leur propre personnage). Hormis quelques rares exceptions, c'est ce dernier aspect qui a retenu la recherche en danse, attachée à montrer comment les qualités d'un danseur lui permettent de «se transformer plastiquement» (Christout, p. 143).

Ces transformations, qui ne sont pas le propre du cinéma, deviennent à son contact tout à fait particulières à cause des propriétés mêmes de l'image cinématographique. La métamorphose, notamment, est facilitée par les qualités de l'image cinématographique, par son pouvoir de faire disparaître et réapparaître des objets. L'absence-présence des objets, leur apparition-disparition est essentiellement le fait de la lumière qui est le propre de l'image-cinéma. Marie-Françoise Grange écrit à ce propos :

\footnotetext{
Pas d'image sans lumière, ni conception, ni réception, pas de perception. Quant à l'image cinématographique, «ce fantôme qu'on ne touche que des yeux» (...) ne se définit-elle pas justement comme l'image-lumière? Le cinéma est histoire de projection d'un faisceau lumineux sur un écran (p. 37).
}

À cet égard, le film Pas de deux de Norman McLaren est exemplaire, car il fait de la lumière l'empreinte qui se moule à des formes (les corps dansants), les incorpore, les fait «vivre et mourir» par une série de déclinaisons des mouvements, de leur dissection sans cesse répétée et déployée, créant dans l'aplat de l'image, de son fond, l'illusion de la troisième dimension. Ce repli et ce dépli de la lumière (du blanc), des corps-formes lumineux désignent le phénomène de clignotement de l'imagelumière :

(...) qui ouvre sur quelque chose de labile, de difficilement nommable, sur ce phénomène interne à la représentation qui relèverait de ce Christine Buci-Glucksmann nomme «ce nœud topologique de la représentation et de l'irreprésentable», de la représentation et «de son excès» (...). Il y a là surgissement $d^{\prime} u n$ autre espace, comme si une limite avait été franchie (Grange, p. 3712 ). 
Il y a là un dépassement qui décale le représenté, qui abstrait (dans les deux sens du terme) sa présence. On l'aura compris, cette capacité transformatrice contenue dans le fait cinématographique, recoupe le procès de la danse, qui fait sortir le sujet de lui-même, le met à l'épreuve de l'autre.

La transfiguration supportée par la lumière est accentuée par le montage, les angles de prises de vue et la position du personnage dansant. Elle est poussée, dans les films sur la danse, à ses limites extrêmes. Par exemple, dans Flash Dance les séquences de danse reproduites par les images-cinéma s'apparentent à celles des vidéoclips : rythmes saccadés des gestes, images flash et battantes qui découpent à outrance les mouvements selon des cadences très accélérées. Le mouvement y est décomposé en unités dont la «monstration» ne suit pas l'ordre logique de leur emplacement à l'intérieur de la chaîne dansée (syntagme). Ces unités sont arrachées à la concaténation intraséquentielle qui forme et fait le mouvement : elles deviennent de purs moments d'«exposition». L'espace scénique est, dans ce contexte, aboli, complètement aplati, dévidé. C'est le moment où le corps dansant est entièrement soumis aux jeux de l'imagelumière, qui devient à son tour, par un effet de renversement, image dansante.

Ces types de métamorphose, qui contribuent aux transformations du corps dansant et de toute figure au cinéma, appartiennent au mécanisme (symbolique) de ces images éblouissantes qui façonnent le champ imaginaire13. Cependant, l'opération de métaphorisation ou d'élaboration de systèmes de similarités qui consiste à remplacer un visage par un autre, une couleur par une autre ou signifier par un son des émotions, ne se situent pas au même degré que l'opération qui définit la deuxième dimension de la métamorphose et cela, même s'il s'agit dans les deux cas de renvoi et même si ces deux opérations ne sont pas exclusives l'une de l'autre.

La deuxième dimension de la métamorphose est dans une certaine mesure plus complexe puisqu'elle concerne la mise du sujet hors de lui-même, vers cet autre auquel aspire la danseuse de Flash Dance, d'où l'allusion notée plus haut à la réfutation du corps. À ce corps s'en substitue un autre (Idéal) auquel le sujet doit raccrocher son désir. Si «danser c'est se prendre soi-même comme objet de son propre désir» (Costelcalde, p. 127), tenter de faire coïncider le corps imparfait avec le corps parfait, cela veut dire "retrouver l'état de la toute puissance (vécue dans la satisfaction hallucinatoire)» (Béreaud, p. 397). Ce corps second pour lequel le corps dansant se contorsionne est ailleurs. Le 
désir de cet ailleurs trouve son illustration dans le film Isadora Duncan au sous-titre bien éloquent : "The Loves of Isadora». Dans ce film, le rapport de la danseuse à la nature, aux mythes grecs et au ciel constitue l'élément essentiel. Isadora enseigne à ses protégés («ses oiseaux» comme elle le dit) à lever la tête vers le soleil, vers le ciel. «Étendez, leur dit-elle, levez les bras. Tout le corps, tendu, tendez également les doigts, tout le corps, les épaules»! Toutes les parties du corps sont mobilisées et s'additionnent pour s'adresser au ciel et le supplier. Le corps d'Isadora est continuellement en position de demande. Deux séquences commencent par la représentation des bras de la danseuse tendus vers le haut. Dans d'autres films l'exhortation s'adresse aux pieds. Ainsi dans Dirty Dancing, l'impératif de ne pas baisser le talon («Don't put your heel down»), qui relève de l'apprentissage de l'équilibre, s'intègre dans la démarche qui consiste à défier les lois de la pesanteur, à les nier.

Le corollaire cinématographique à ce mouvement vers le haut est l'utilisation de la plongée, parfois même de la plongée verticale, ce regard radical, surplombant de la caméra qui saisit totalement le tracé chorégraphique du corps dansant (cf. Isadora Duncan ou Flash Dance).

Introduite par Busby Berkeley (1933), soucieux de reproduire l'ensemble des figures chorégraphiques, la plongée verticale est à la fois enregistrement des prouesses des corps 14 et indication du lieu d'où on regarde. Et c'est du ciel que regarde la caméra. L'assimilation du regard de la caméra et du regard de Dieu a été notée par de nombreux chercheurs en études cinématographiques 15 . Cependant, on n'a pas tiré toutes les conséquences de cette remarque, notamment celle qui conçoit que «toutes ces formes du donné à voir» installent le cinéma dans l'ordre du mythe. En effet, le cinéma n'exclut pas, au nom de sa modernité, la captation et la relation identificatoire qui règlent les liens des individus aux sociétés; dans ce sens il est dans la continuité de la pratique des masques dans les sociétés nonoccidentales. Il révèle la puissance des images dans le procès d'assujetissement des individus, leur production en sujets. Ce pouvoir s'accentue lorsqu'il convoque simultanément le corps et l'image.

Par ailleurs, la jointure des regards qui se constitue à travers la multiplicité des points de vue (les angles de prise de vue, la caméra subjective, etc.) et sur laquelle se décalquent les regards de la caméra et du spectateur du film, est redoublée et répétée de façon quasi obsessionnelle par la présence des personnages spectateurs dans le film. Les films qui me servent d'exemples 
mettent tous en scène, avec des degrés plus ou moins complexes, cette enfilade des yeux et cette représentativité (public, chorégraphe, producteur ou tout simplement danseurs et danseuses - Carmen constitue à cet égard un exemple privilégié). Nonobstant les convergences ou les divergences de regards entre ces deux types de spectateurs (du et dans le film), ces mises en scène qui insistent sur la présence du spectateur dans le film mais qui limitent son regard et le restreignent posent le corps-dansant dans sa relation au spectacle, le rendent spectaculaire. Elles le «mettent en représentation» et l'exposent aux regards. Cette exposition est largement renforcée et soutenue par la spécificité narrative des films (les films sur la danse sont des mises en abyme, des spectacles dans le spectacle).

Quant aux spectateurs du film, je crois que la notion de «vantage point» (point avantageux) qu'utilise Jacques Aumont (p. 70) signale un élément important de ce cérémonial. En effet, produire les points de vue les plus efficaces, des points de vue "sans rival», montrer les gestes de tous les côtés, sous tous les angles, c'est produire l'état jubilatoire de l'objet enfin entièrement maîtrisé. C'est là que le spectateur du film se démarque du spectateur dans le film, car la caméra envahit (pour lui) l'espace scénique de la danse, le transfigure et présente les mouvements du corps dansant dans un alignement de plans qui s'ajuste et obéit à leur déroulement (l'utilisation des raccords sur le mouvement - très nombreux dans Flash Dance; les changements des angles de prise de vue et d'échelles de plans; les déplacements de la caméra, ses glissades et ses poursuites - la caméra suit frénétiquement, dans Soleil de nuit, le déplacement du danseur et est littéralement collée à ses talons). Ce travail de la caméra, d'une part confère une place privilégiée au spectateur du film qui, contrairement au spectateur dans le film, n'est pas rivé à sa place et d'autre part, grâce aux vertus de la lumière, crée l'illusion du proche en gardant le spectateur le plus près possible, dans l'intimité du danseur. L'organisation de la mise en mouvement, qui va des plans séquences qui englobent d'importants segments chorégraphiques 16 aux plans courts et hachés qui découpent ces segments, fait en sorte que le spectateur danse lui aussi, qu'il jouit de cette jouissance du corps dansant à un tel degré qu'il en arrive à oublier, si ce n'est à réfuter, son propre corps dont la motricité, on le sait, est déjà fortement réduite.

Cette réfutation a pour objet le corps anatomique qui, par un paradoxe troublant, devient vide de sens, suppléé qu'il est par ce corps second, sujet du savoir (de tout savoir), auquel 
protagoniste-danseur et spectateur tentent désespérément de s'identifier, car la danse, comme le cinéma, offre le corps idéal, désespérément et sérieusement, puisque le rire est écarté dans cette opération et parfois même le sourire : "Don't smile! It's not a high school play», dit Joe, le chorégraphe de All That Jazz (on le comprend, il danse avec la mort).

\section{Du dressage et de l'organisation}

En recourant au rire, je souhaite introduire une autre dimension de la fabrication du corps dansant qui est à rapprocher de la conception de la danse en tant que relais symbolique. Cette dimension concerne le travail de dressage et de modelage du corps qui suppose la position verticale (la position debout), dont on dit qu'elle est la quintessence de l'être humain17. La barre est un des instruments qui sert à la recherche de l'équilibre, de la rectitude et de la maîtrise du corps (dans les sens de maîtrise du corps et de corps maîtrisé) enseignée en danse. On croirait à tort que la danse moderne y a renoncé. Interviewé à l'occasion d'un spectacle qu'il présentait à Berlin au printemps 1989, Maurice Béjart, un des chantres de cette danse, répond : «Pour nous danseurs, la loi c'est la barre». Cependant, cet acte de foi s'inscrit sous le signe d'une ambiguïté dans certains films de notre corpus. Dans Flash Dance, si la barre occupe une partie du loft de la protagoniste, elle est rejetée par elle, qui rêve néanmoins de devenir danseuse de ballet. Cette danseuse de bar méprise les danseurs classiques produits par l'académie même à laquelle elle désire avant tout s'intégrer, s'incorporer. Mais ce paradoxe, semblable à celui du pauvre qui aime la richesse et déteste les riches, n'est en fait qu'apparent parce que la barre répond à l'imposante architecture classique de l'académie de danse, écrasante par sa grandeur et son histoire séculaire (cf. la contre plongée accentuée du bâtiment lorsque la jeune fille le regarde pour la première fois).

La finale du film est assez significative sur ce point, non parce que la danse "profane» (apprise sur le tas, même dans la rue) exécutée par la jeune femme lors de son audition soigne l'académisme de sa sclérose et le libère, mais parce qu'elle montre comment ce dernier sait intégrer et canaliser les éléments étrangers qui, il faut insiter là-dessus, touchent à la forme et non au fondement ${ }^{18}$. La barre est toujours là, même si elle n'est pas montrée19. Elle s'ajoute, dans le cas de la danse, à ce que Georges Vigarello nomme «les panoplies redresseuses», qui vont au delà de leur aspect instrumental pour rejoindre les préceptes de la pédagogie du maintien et des civilités. La barre fait partie intégrante de la recherche de la rectitude qui caractérise 
l'Occident. Or, cette recherche a repris dans tous les domaines, y compris dans celui de la gymnastique, le modèle de l'armée, la position droite du soldat. Pierre Legendre voit une similitude entre la chorégraphie militaire et celle de la danse et l'intègre dans le rapport de soumission du corps aux institutions (pp. 118120). Outre la similitude que peut repérer la démarche analytique entre la danse et l'art militaire, l'histoire des mentalités nous apprend que ces domaines se sont mutuellement influencés. Ainsi, dans la société de cour où le critère de l'élégance prédomine :

La danse est exercice de maintien et de perfectionnement de l'attitude. Elle est ce qui dénoue (...). Dans un tel contexte la création en 1661 de l'Académie royale de danse, régentant entre autre la formation des maîtres, confirme le prestige institutionnel d'un art (...) 20 reconnu comme l'un des plus honnêtes et des plus nécessaires à former le corps et à lui donner les premières et les plus naturelles dispositions à toutes sortes d'exercices et entre autres à ceux des armes (Vigarello, p. 53).

«Le corps redressé» 21 est un corps dressé, une chair à modeler soumise patiemment aux exercices quotidiens, aux répétitions harassantes, au ciselage méthodique. La chorégraphie consiste à organiser un ensemble de figures à partir du corps, à justifier le geste à faire, à le réctifier, à trouver le lieu adéquat (même dans l'improvisation), à modeler le corps afin de le rendre apte à signifier. Pour y parvenir, il faut payer de son corps (anatomique).

Tous les films, sans exception, montrent des danseurs en répétition. Qu'ils manipulent les machines aérobiques (Flash Dance) ou qu'ils s'efforcent d'apprendre des tours de voltige dans les eaux d'un lac (Dirty Dancing) ou encore qu'ils s'exposent au regard marchand ou à celui du maître (All That Jazz, Carmen), tous ces corps, sans distinction de race, de culture, de danse ou de sexe, travaillent en suant. Il n'est pas facile d'apprendre à faire bouger (construire) le corps idéal, surtout lorsqu'on s'évertue à enclaver la danse dans le spectacle et à l'éloigner de ce que les danses des cultures non occidentales reconnaissent explicitement : la transe. Pour ces cultures non occidentales, entrer en transe c'est bien entrer en contact avec un représentant suprême. La volonté de confectionner un autre corps tend à décharner le corps anatomique, à le dépouiller de sa chair. Ce travail de la danse est accentué par celui de l'image cinématographique qui reproduit uniquement des corps d'ombre et de lumière (d'autres diront des corps de morts-vivants). De nombreuses correspondances, on l'a vu, lient l'art 
chorégraphique et l'art cinématographique, correspondances de symétrie entre le système géométrique très élaboré et très précis de la danse, de l'ordre du soulignement, du tracé (la graphie) et sa captation par une caméra tout aussi déterminée par des règles géométriques, repérables dans les cadrages, les angles de prises de vue, les axes, les mouvements combinés, les frôlements, les enveloppements, etc. Cette combinaison danse-cinéma, dans laquelle la musique est fondamentale (pensons à l'attribution circonstanciée de «musicale» à la comédie musicale des années 30 et 40) est largement actualisée par les règles et les notations de la danse. Parce qu'ils réfèrent à elle, les films contemporains sur la danse suscitent deux types de fascination : celle produite par l'image cinématographique et celle produite par la danse, le corps dansant plus exactement.

Cette double fascination a lieu en dépit des découpages intempestifs qui décomposent le corps et le livrent en morceaux. Le film sur la danse, comme avant lui la comédie musicale, met donc en jeu la problématique de l'ordonnancement des corps, de leur organisation. On serait en effet tenté de croire, parce qu'elle mobilise souvent un grand nombre de danseurs, que la comédie musicale est plus à même d'afficher le travail d'organisation, de composition, de coordination des danseurs et des arrangements kaléïdoscopiques. Or le procédé chorégraphique est tout aussi prégnant dans les films sur la danse, même s'il paraît moins immédiat et moins évident. Les modalités d'occupation de l'espace, les combinaisons des corps, qu'ils rassemblent des couples ou décrivent les replis et les étirements d'un corps unique, sont en effet strictement réglés.

\section{De la beauté du corps dansant}

Les compositions chorégraphique et cinématographique procurent à profusion ce qui satisfait le regard : la Beauté du corps. Cependant on aura compris d'après ce qui vient d'être développé, que la Beauté, le beau corps du danseur et de la danseuse dépend d'une codification qui relève elle-même d'un discours voulant instituer le corps parfait. Le ravissement, au sens religieux du terme (transport vers le ciel) ressenti au spectacle de la danse, s'appuie sur ces images cinématographiques saisissantes, admirablement imbriquées et centrées sur le corps. On peut parler dans ce cas de fulgurance. La Beauté s'articule à l'ostentation de la caméra qui se faire remarquer. Elle est entretenue par l'adhérence entre les mouvements de la caméra et les mouvements du corps dansant, qui trouve son point limite dans la vitesse d'exécution. 
Outre qu'elle est rattachée aux codes de la composition chorégraphique et cinématographique, la Beauté se rattache au propre et son corollaire la souillure, comprise ici dans son acception première de pollution sexuelle. Je prendrai l'exemple du film Dirty Dancing 22 dont le titre français, Danse lascive, traduit un glissement très significatif.

Ce film met en place le schéma d'une division entre, d'un côté la danse sociale et correcte ou bien les sautillements infantiles et sans conséquences, et de l'autre la danse des Tropiques. La danse de bon ton pratiquée par les familles de riches vacanciers s'oppose à celle de la faune de jeunes danseurs et danseuses qui loin des regards, se laissent aller à la «lascivité». Baby, c'est le prénom de la jeune ingénue qui s'aventure dans les eaux vaseuses et interdites, nous fait découvrir un univers où les corps des jeunes couples s'épanchent aux rythmes de la rumba, du méringué, etc. Son apprentissage (elle ne porte pas ce prénom pour rien) consistera, au fil du récit, à dévider ces danses de leur signification. Ce qu'étaient ces imbugadas, ces sambas, ces cuecas, signifiant littéralement «ces heurts de nombrils» (Bastide, p. 185) se trouvent, dans le film, progressivement réappropriées pour devenir de simples danses socialisées.

La récupération de ces danses ne se réalise pas seulement à la fin du film, c'est-à-dire au moment où Baby peut enfin pratiquer avec son compagnon, enfin accepté par son père, sa danse lascive, toute ordonnée, sous l'œil charmé des spectateurs qui ne tarderont d'ailleurs pas à leur emboîter le pas, elle commence bien avant puisqu'elle est l'objet du film. Cette appropriation, qui est aussi une opération de nettoyage, se fait sur le travail de décomposition des gestes, dans leur recomposition chorégraphique. Ce qui était supposément «la danse des nombrils", ce qui était lié à la proximité des corps se transforme, par la force d'un ordonnancement didactique (pédagogique), en une simple indication de distance : "That's my dance space, that's your dance space. I don't want yours, you don't want mine», dit le jeune et séduisant maître à Baby. L'apprentissage et la décomposition des gestes servent à rassurer à propos d'un danger qui n'en est pas véritablement un. Ce leurre explique le finale où le couple peut danser et se faire admirer en toute légalité. Le déroulement du récit, la segmentation des gestes et leur mémorisation (par l'élève Baby) dissolvent le potentiel menaçant et «polluant» de la danse pratiquée par les jeunes danseurs et danseuses. Ils l'intègrent dans l'acceptable 23 aurour duquel se cristallise progressivement l'idée du beau corps, l'idée de la Beauté (de la danse). 
La beauté est une construction esthétique et, par là même, une construction politique, au sens de mise en ordre des éléments qui la composent. Elle a partie liée avec l'ordre du social et du politique. La beauté du corps dansant quadrille les zones à éclairer, à supporter théoriquement et blanchit les zones d'ombre, le sale et l'abject. Le dirty du dancing qui est annoncé dans le titre est désamorcé par le travail du récit, aussi bien sur le plan figuratif que sur ceux de l'action et de l'événement.

La Beauté participe dès lors à ce qui se fabrique politiquement et enferme le corps dans le cycle des répétitions des fictions qui le font tourner, cinématographiquement et chorégraphiquement, pour mieux signifier la Loi. Cette remarque revient à ce que j'avançais au début de cet article, à savoir l'intégration de la danse dans le travail de fabrication du social.

J'ai insisté dans ce texte sur la nécessité qu'a la société de se représenter à travers des relais symboliques, dont la danse (le corps dansant) et l'image qui appellent au spirituel et à la liberté. $\mathrm{Ce}$ serait oublier que «l'auto-suffisance du danseur (...) est traversée de part en part, organisée totalement par un idéal collectif (...) et qu'il s'opère en elle "la capture sociale et politique de l'idéal narcissique du Sujet"» (Costelcalde, p. 128). Cet aspect est renforcé par le cinéma dont le dispositif inclut le sujet et le procès de la subjectivité. La démarche empruntée ici contribue à repenser ou à réinterroger la propension à considérer les films sur la danse selon le strict point de vue esthétique, dépouillé de son contenu politique, et adhère aux arguments de Marcia Butzel qui écrit :

\begin{abstract}
More than any another kind, dance film continues to be defined exclusively in terms of its physical and technical properties as an aesthetic object. Instead, it must be recognized as part of the historical process of meaning production we call culture, inflecting and inflected by social, political, and economical processes as well as an aesthetic and representational ones (p. 113).
\end{abstract}

L'analyse de quelques films sur la danse montre que le corps est bien un lieu d'organisation des savoirs, et que le corps dansant au cinéma réfère à autre chose qu'à la forme «tangible» qu'il représente. Ce procès de renvoi est au carrefour des présupposés de la danse et du cinéma en tant que dispositifs imaginaires. Le cinéma rencontre la danse en redoublant et en ritualisant son idéal du beau, c'est-à-dire l'ordonnancement esthétique et politique des corps, et par là, il est au centre de la production du sens.

Université Laval 
1 Je remercie Yvan Simonis, Marc Vernet et Heinz Weinmann pour leurs commentaires sur une première version de ce texte. Je reste cependant seule responsable des limites de son contenu.

2 Rick Altman, The American Film Musical (Bloomington \& Indiana : Indianna University Press, 1987).

3 Lucy Fischer, «The Image of Woman as Image : The Optical Politics of Danses», Genre: the Musical (London, Boston and Henley : Routhledge \& Kegan Paul, 1981).

4 Les idées développées ici doivent beaucoup aux travaux de Pierre Legendre qui remettent en cause l'empirisme «ontologique» entourant les définitions de ce qu'on appelle «la société». Pour parler de la société et de ce qui l'active («la fait tourner»), l'auteur introduit le terme de «fiction». «Il s'agit, dit-il, en toute société de construire le Sujet monumental de fiction qui puisse tenir un discours fondateur et produire l'effet normatif». Pierre Legendre, «Le Ficelage institutionnel de l'humanité», Anthropologie et sociétes, vol. 13, no 1(1989) p. 65. Nous sommes dès lors, comme l'écrit Yvan Simonis, «dans l'art (...) du leurre nécessaire, dans la ruse de la guerre des institutions pour dresser le sujet à être humain, dans l'arbitraire de ce dressage par le Pouvoir». "Notes critiques sur le droit et la généalogie chez Pierre Legendre», ibid., p. 54. Je souligne.

5 Victor Turner souligne cette ambivalence constitutive en écrivant que «le moment dans et hors du temps» est celui de la «célébration du rituel». Les Tambours de l'affliction. Analyse des rituels chez les Ndembu de Zambie (Paris : Gallimard, 1972) p. 12.

6 Contrairement au cinéma, la danse échappe très rarement à un discours quasi mystique qui l'inscrit dans dans le subversif et la propulse dans une sorte de transcendance ineffable. Elle est ainsi l'expression par excellence de la spiritualité, selon Roderyk Lange, «un indicatif de la transcendance», selon Roger Garaudy, et tresse un lien innarrable et privilégié avec «l'infini», selon Andrée Martin. Ces caractéristiques que l'on soupçonne à peine dans les œuvres cinématographiques, ou du moins qui sont repérables seulement dans certains de leurs thèmes ou de leur formes, semblent constituer en revanche l'essence de la danse. Voir à ce sujet Roderyck Lange, The Nature of Dance. An Anthropological Perspective (London : Mac Donald\& Evans, 1975) p. 55, Roger Garaudy, Danser sa vie (Paris : Seuil, 1973) p. 24 et Andrée Martin, «La danse de cinéma», Protée, vol. 19, no 3 (1991) p. 75.

7 Willy Apollon, «Le Père et le pire», La Famille. Enjeux et impasses, colloque du Goupe Interdisciplinaire Freudien de Recherches et d'Interventions Cliniques et Culturelles, Québec, novembre 1991. Communication inédite.

8 Willy Apollon, «La reproduction de la subjectivité se fait par la transmission de la limite», idem.

9 Je dois cette idée à Yvan Simonis que je cite de mémoire. Les récits des mythes fondateurs des sociétés, leur existence même, sont à ce titre très révélateurs des tentatives de «saisissement» du point d'origine du social.

10 Les italiques sont de moi.

11 Dans cette partie, mon approche du savoir s'inspire essentiellement de la psychanalyse pour laquelle «la méconnaissance est une fonction» (Notes d'une conférence de Pierre Legendre donnée à L'Université Laval en novembre 1989). Cette fonction joue au même titre que le savoir en investissant tous les discours, y compris ceux qui semblent s'en écarter, 
comme par exemple le discours scientifique. Dans les propos qui suivent, Daniel Sibony montre bien comment on est pris dans l'articulation connaissance / méconnaissance et pourquoi elle est fondamentale : «Il suffit d'entendre la voix de celui qui tient un discours scientifique, et de le regarder (ou il suffit de tenir soi-même un tel discours et de s'en décaler), pour ne jamais douter de ceci que sur quoi on y fait silence et qui touche à l'énonciation, loin de fonctionner comme ce qu'il apparaît - un point aveugle qui résume une étendue uniforme - est au contraire traversé par les singularités et les tressaillements du désir et de libido tels qu'ils forment l'économie libidinale d'un sujet et sa réplique dans le lien social. Et que ce soit sur ce semblant ou cette méconnaissance que ça marche, est lourd de conséquences dans la manière dont la culture est marquée par la Science et le désir par le savoir». Le Nom et le corps (Paris : Seuil, 1974) p. 56.

12 Les italiques sont de moi.

13 Voir à ce sujet Christian Metz, Le Signifiant imaginaire (Paris : Christian Bourgeois, 1977).

14 Lucy Fischer note que les corps dansants apparaissent comme étant tous identiques dans les films de Busby Berkeley, et montre comment la similarité se transforme rapidement en identite : «Part of the humor of the numbers, in fact, arises from our perception that the women look remarkably alike. This notion is eventually catapulted into another realm entirely in "I Only Have Eyes for You". What happens in most Berkely numbers (and quintessenticilly in "Dames") is that the women lose their individuality in a more profound sence than through the similarity of their physical appearance». «The Image of Woman as Image: The Optical Politics of Dances», Genre: the Musical (London, Boston and Henley : Routhledge \& Kegan Paul, 1981) pp. 74-75.

15 Voir l'excellent chapitre que Jacques Aumont consacre au regard, dans son livre L'Eil interminable. Cinéma et peinture (Paris : Séguier, 1989).

16 A ce sujetCarmen est exemplaire, car les découpages en plans courts y sont très rares et sont remplacés par des plans séquences qui capturent l'entièreté des gestes.

17 Pierre Merle, L'Homme et la verticale (Paris : La Colombe, 1960).

18 C'est pourquoi je ne fais pas de distinction entre les différents types de danse; elle est considérée ici dans un sens générique.

19 Je ferai abstraction du volontarisme à outrance, du «self made woman» qui recompense, du thème de l'incompréhension et du pseudo-triomphe de la culture populaire, qui mériteraient qu'on s'arrête longuement.

20 Vigarello cite, dans ses propos, un extrait des lettres patentes pour l'établissement de l'Académie royale de danse, mars 1661.

21 Titre du livre Georges Vigarello dont cette partie s'inspire fortement.

22 Même s'ils jouent sur le registre de la liberté et la nécessité d'une expression sans entrave (entendons idéologique), les films comme Soleil de nuit ou Isadora Duncan ne soulèvent pas moins, eux aussi, cette question de la pureté.

23 Marc Vernet dirait Le bien, lui qui note dans les films américains des années 40 une trajectoire similaire de l'incription de la figure de l'idéal( $e$ ). Selon lui, «L'idéal(e) peut servir de révélateur des défauts et des petitesses, mais c'est pour en faire ressortir la tendre humanité qui doit moins chercher à les masquer qu'à les accepter et si possible en tirer parti pour faire le bien». Figures de l'absence. De l'invisible au cinéma (Paris : Cahiers du cinéma, 1988) p. 124. Rick Altman le montre également pour le cas de la comédie musicale qu'il analyse comme une tentative de rédemption face au sentiment de 
perte des valeurs fondamentales qui prévalaient dans la société américaine de la même période. Les artifices de ce genre réussirent le doublet spectaculaire de redorer l'industrie cinématographique américaine au bord de la ruine, et de miser, en usant de la légéreté des thèmes et des corps, sur le recentrement des idéaux de la société américaine, sur sa moralité. The American Film Musical (Bloomington \& Indiana : Indianna University Press, 1987).

\section{BIBLIOGRAPHIE}

Aubertin, S., et al. «L'Image de la danse dans la publicité télévisée». La Recherche en danse 3 (1984) pp. 83-96.

Aumont, J. L'Eil interminable. Cinéma et peinture. Paris : Séguier, 1989.

Bastide, R. Les Amériques Noires. Les Civilisations africaines dans le Nouveau Monde. Paris : Payot, 1967.

Bédard, Yves. «Images technologiques : ce qu'il advient de la mémoire». Cinémas vol. 1, no 3 (1991) pp. 89-101.

Bellour, R. «Le Regard de Haghi». Iris (Cinéma et narration I) (1986) pp. 513.

Béreaud, J. «Sociologie de la connaissance et psychanalyse : autour du concept de représentation». Cahiers internationaux de sociologie, vol. LXXXIII (1987).

Butzel, M. «Paradigms for cognitivism? : The Perception of Movement and Film Choreography». Iris 9 (Cinema and Cognitive Psychology) (1989).

Charles, A. «Flux de marche avec piétinement». Traverses 14-15 (1979) pp. 81-92.

Christout, M.-F. «Chorégraphie». Encyclopaedia Universalis 5 (1989) pp. 738-739.

Christout, M.-F.. «Danse et mime, actes de la métamorphose». Corps écrit 26 (1986).

Costelcade, N. «Fonction de l'image féminine dans la danse : une hypothèse». La Recherche en danse 1 (1982).

Gil, J. Métamorphoses du corps. Paris : La Différence, 1985.

Grange, M.-F. «Effet toile. Sur une séquence de Mauvais sang (Léos Carax, 1986)». Protée, vol. 19, no 3, 1991.

Hamon, P. Texte et idéologie. Paris : PUF, 1984.

Hanna, J.-L. Dance, Sex and Gender, Signs of Identity, Dominance, Defiance, and Desire. Chicago, London : The University of Chicago Press, 1988.

Hanna, J.-L. To Dance is Human. In John Blacking (direction), Anthropology of the Body. London : Académic Press, 1977, pp. 211232.

Larisse, F. «"Art Press" et L'Avant-Garde en danse». La Recherche en danse 3 (1984) pp. 75-80.

Legendre P. «Le danseur : un instrument dans la main du chorégraphe? Note sur la danse considérée comme mouvement politique». Le Désir politique de Dieu. Paris : Fayard, 1988, pp. 197-199.

Legendre, P. La Passion d'être un autre. Étude pour la danse. Paris : Seuil, 1978. 
Lifar, S. La Danse académique et l'art chorégraphique. Paris : Gonthier, 1965.

Mauriès, P. «Royaume du Tropique. Petite mythologie de la disco». Traverses 18 (1981) pp. 105-110.

Melon, M.-E. "Quand les fantômes viennent à notre rencontre». In Philippe Dubois et Yves Winkin (direction), Rhétorique du corps. Bruxelles, Paris : Éd. Universitaires \& De Boek / Université, 1988, pp. 183-201.

Pujade-Renaud, C. Danse et narcissisme en éducation. Paris : E.S.F., 1976.

Segra Decron, G. "À propos de la danse-thérapie : L'Image du corps, laquelle?». La Recherche en danse 3 (1984) pp. 161-170.

Tournay, M. «Espace scénique, espace chorégraphique». La Recherche en danse 1 (1982) pp. 103-110.

Vernant, J.-P. «La Catégorie psychologique du double». Mythe et pensée chez les Grecs II. Paris : Maspéro, 1965, pp. 65-78.

Véron, E. «Discursivités de l'image». L'image fixe. Espace de l'image et temps du discours. Paris : Centre Georges Pompidou, 1983, pp. 116138.

Vigarello, R. Le Corps redressé. Paris : Jean-Pierre Delarge, 1978.

Williams, D. «Semasiology. A Semantic Anthropological View of Human Movements and Actions». In D. Parkin, Semantic Anthropology. London: Academic Press, 1982, pp. 160-181. 